\title{
Vascular Endothelial Growth Factor Receptor-3 (VEGFR-3): A Marker of Vascular Tumors with Presumed Lymphatic Differentiation, Including Kaposi's Sarcoma, Kaposiform and Dabska-Type Hemangioendotheliomas, and a Subset of Angiosarcomas
}

Andrew L. Folpe, M.D., Tanja Veikkola, M.D., Reija Valtola, M.D., Sharon W. Weiss, M.D. Department of Pathology, Emory University (ALF, SWW), Atlanta, Georgia, and Molecular/Cancer Biology Laboratory, University of Helsinki (TV, RV), Helsinki, Finland

Recently, a novel monoclonal antibody to vascular endothelial growth factor receptor 3 (VEGFR-3), a tyrosine kinase receptor expressed almost exclusively by lymphatic endothelium in the adult, has been shown to react with a small number of cases of Kaposi's sarcoma (KS) and cutaneous lymphangiomas. We sought to extend these studies to a large number of well-characterized vascular neoplasms to evaluate diagnostic uses of this antibody and to determine whether it defines them in a thematic fashion. Formalin-fixed, paraffin-embedded sections from 70 vascular tumors were immunostained with antibodies to VEGFR-3 von Willebrand factor (vWF), and CD31. Anti-VEGFR-3 was positive in 23 of 24 KS, 8 of 16 angiosarcomas (AS), 6 of 6 kaposiform hemangioendotheliomas, 4 of 4 Dabska tumors, and 2 of 13 hemangiomas. Positively staining angiosarcomas were characterized either by a prominent lymphocytic component, a hobnail endothelial cell similar to that encountered in the Dabska tumor, or spindled areas resembling KS. No VEGFR-3 expression was noted in any cases of epithelioid hemangioendothelioma, pyogenic granuloma, littoral angioma, or stasis dermatitis. vWF expression was seen in 10 of $13 \mathrm{KS}$; 13 of 14 AS; 4 of 5 kaposiform hemangioendotheliomas; and all Dabska tumors, hemangiomas, lymphangiomas, epithelioid hemangioendotheliomas, vascular malformations, stasis dermatitis, and splenic littoral angiomas. CD31 expression was present in 12 of 13

Copyright () 2000 by The United States and Canadian Academy of Pathology, Inc.

VOL. 13, NO. 2, P. 180, 2000 Printed in the U.S.A.

Date of acceptance: August 25, 1999.

Address reprint requests to: Andrew L. Folpe, M.D., Department of Pathology, H-180, Emory University Hospital, 1364 Clifton Road NE, Atlanta, GA 30322; e-mail: afolpe@emory.edu; fax: 404-712-4454.
KS, 13 of 14 AS, and in all other cases. Expression of VEGFR-3 is a very sensitive marker of KS, kaposiform, and Dabska-type hemangioendotheliomas, suggesting that all show at least partial lymphatic endothelial differentiation. Expression of VEGFR-3 does not reliably discriminate KS from AS. However, the expression of VEGFR-3 by certain AS having Kaposi-like areas, a prominent lymphocytic infiltrate, or hobnail endothelium may define subset(s) having phenotypic, if not pathogenetic and biologic, differences.

KEY WORDS: Angiosarcoma, Kaposi's sarcoma, Lymphatics, Vascular endothelial growth factor receptors.

Mod Pathol 2000;13(2):180-185

The line of differentiation of Kaposi's sarcoma (KS) has been long debated, with arguments offered in favor of endothelial, smooth muscle, or undifferentiated mesenchymal lineage. There is now overwhelming evidence that KS shows endothelial differentiation (1-8) even though there is no consensus on whether the cells of KS have a lymphatic $(2,8)$ or vascular endothelial $(1,7,9)$ phenotype. Recently, a novel monoclonal antibody to vascular endothelial growth factor receptor 3 (VEGFR-3), a tyrosine kinase receptor expressed almost exclusively by lymphatic endothelium in the adult, has been shown to react with a small number of cases of KS (4) and cutaneous lymphangiomas (10). We sought to extend these studies to a large number of well-characterized vascular neoplasms to evaluate possible diagnostic uses of this antibody because a highly sensitive and specific antibody for KS would clearly be a useful diagnostic. At present, antibodies to CD31, CD34, and von Willebrand factor (vWF, Factor VIII related antigen) are 
commonly used in the distinction of KS from nonvascular neoplasms, but they suffer from lack of sensitivity, in the case of vWF and CD31 $(5,6,11)$ and lack of specificity, in the case of CD34 (12-14). Furthermore, they are of little use in distinguishing KS from benign and malignant vascular tumors $(7,8)$. In addition, we wanted to determine whether VEGFR-3 defines other vascular lesions in a thematic fashion.

\section{METHODS}

Formalin-fixed, paraffin-embedded sections from 70 vascular tumors (24 KS, 16 angiosarcomas (AS), 13 hemangiomas, 6 kaposiform hemangioendotheliomas, 3 intra-abdominal lymphangiomas, 4 malignant endovascular papillary angioendotheliomas [Dabska tumor], 1 epithelioid hemangioendothelioma, 1 splenic littoral angioma, 1 vascular malformation, and 1 case of stasis dermatitis) were retrieved from the consultation files of one of the authors (SWW) and the archives of the Departments of Pathology of Emory University and the University of Washington Medical Center. None of the AS were known to be associated with chronic lymphedema or prior irradiation. Deparaffinized sections were immunostained with a monoclonal antibody raised against VEGFR-3 (9D9F9, 1:1000; laboratory of Dr. K. Alitalo). The production of this antibody has been described in detail elsewhere (4). A subset of cases were also immunostained with antibodies to vWF (F8/86, 1:100; Dako Corp., Carpinteria, CA) and CD31 (JC70, 1:80; Dako). Negative controls consisted of omission of the primary antibody. Sections were subjected to heat-induced epitope retrieval, using a vegetable steamer. Antigens were localized using an avidin-biotin method with 3,3'-diaminobenzidine as a chromogen.

\section{RESULTS}

As detailed in Table 1, strong immunoreactivity for VEGFR-3 was seen in 23 of 24 cases of KS (96\%; Fig. 1). Strong VEGFR-3 expression was also noted

TABLE 1. Immunohistochemical Findings

\begin{tabular}{lccc}
\hline \multicolumn{1}{c}{ Diagnosis } & VEGFR-3 & vWF & CD31 \\
\hline Kaposi's sarcoma & $23 / 24$ & $10 / 13$ & $12 / 13$ \\
Angiosarcoma & $8 / 16$ & $12 / 14$ & $13 / 14$ \\
Kaposiform & $6 / 6$ & $4 / 5$ & $5 / 5$ \\
$\quad$ hemangioendothelioma & & & \\
Dabska tumor & $4 / 4$ & $3 / 4$ & $4 / 4$ \\
Hemangioma & $2 / 13$ & $4 / 4$ & $4 / 4$ \\
Lymphangioma & $1 / 3$ & $\mathrm{ND}$ & $\mathrm{ND}$ \\
Epithelioid hemangioendothelioma & $0 / 1$ & $1 / 1$ & $1 / 1$ \\
Vascular malformation & $1 / 1$ (focal) & $1 / 1$ & $1 / 1$ \\
Stasis dermatitis & $0 / 1$ & $1 / 1$ & $1 / 1$ \\
Splenic littoral angioma & $0 / 1$ & $\mathrm{ND}$ & $\mathrm{ND}$ \\
\hline
\end{tabular}

VEGFR-3, vascular endothelial growth factor 3; vWF, von Willebrand factor; ND, not done. in 8 of 16 (50\%) cases of AS; of the positive cases, six had either a prominent lymphocytic infiltrate or hobnail endothelial cells (Figs. 2 and 3). VEGFR-3 was also expressed in all cases of kaposiform hemangioendothelioma and Dabska tumor (Fig. 4). Two of 13 hemangiomas (15\%) and 1 of 3 cases of intra-abdominal lymphangioma also contained positive cells. The one vascular malformation studied was focally positive, in an area of thin-walled vessels believed to be morphologically very suggestive of lymphatic differentiation.

No VEGFR-3 expression was noted in any cases of epithelioid hemangioendothelioma, littoral angioma, or stasis dermatitis. Positive internal controls (normal lymphatic channels) were present in all cases.

vWF expression was seen in 10 of 13 cases of KS (77\%); 12 of 13 cases of AS (92\%); 4 of 5 kaposiform hemangioendothelioma (80\%); and all cases of Dabska tumor, hemangioma, lymphangioma, epithelioid hemangioendothelioma, vascular malformation, stasis dermatitis, and splenic littoral angioma studied. CD31 expression was present in 12 of 13 cases of KS (92\%), 13 of 14 cases of AS (93\%), and all other studied cases.

The one case of KS that was VEGFR-3 negative was also negative for vWF but was strongly CD31 positive. One case of KS was VEGFR-3 positive but negative for both vWF and CD31. Six of the eight VEGFR-3-positive AS were also strongly positive for both vWF and CD31; one case was vWF negative but strongly CD31 positive, and in one case there was focal and weak vWF expression and strong CD31 expression.

\section{DISCUSSION}

The platelet derived growth factor (PDGF) family, including vascular endothelial growth factor (VEGF) and the closely related molecules VEGF-B, VEGF-C, and VEGF-D, play a significant role in angiogenesis and vascular permeability (15). VEGF-C plays a critical role in lymphangiogenesis; in transgenic mice, VEGF-C has the ability to induce both lymphatic endothelial proliferation and lymphatic vessel formation $(16,17)$. In the adult, expression of the mRNA of the VEGF-C receptor, VEGFR-3 (FLT4), is limited almost exclusively to lymphatic endothelium, although it may be detected in other endothelia at earlier stages of development (18).

Recently, the production of a monoclonal antibody to VEGFR-3, 9D9F9, was reported by Jussila and co-workers (4), who found it to react with lymphatic endothelium but not with capillaries or lymph node high endothelial venules. These authors also found VEGFR-3 expression in paraffin- 


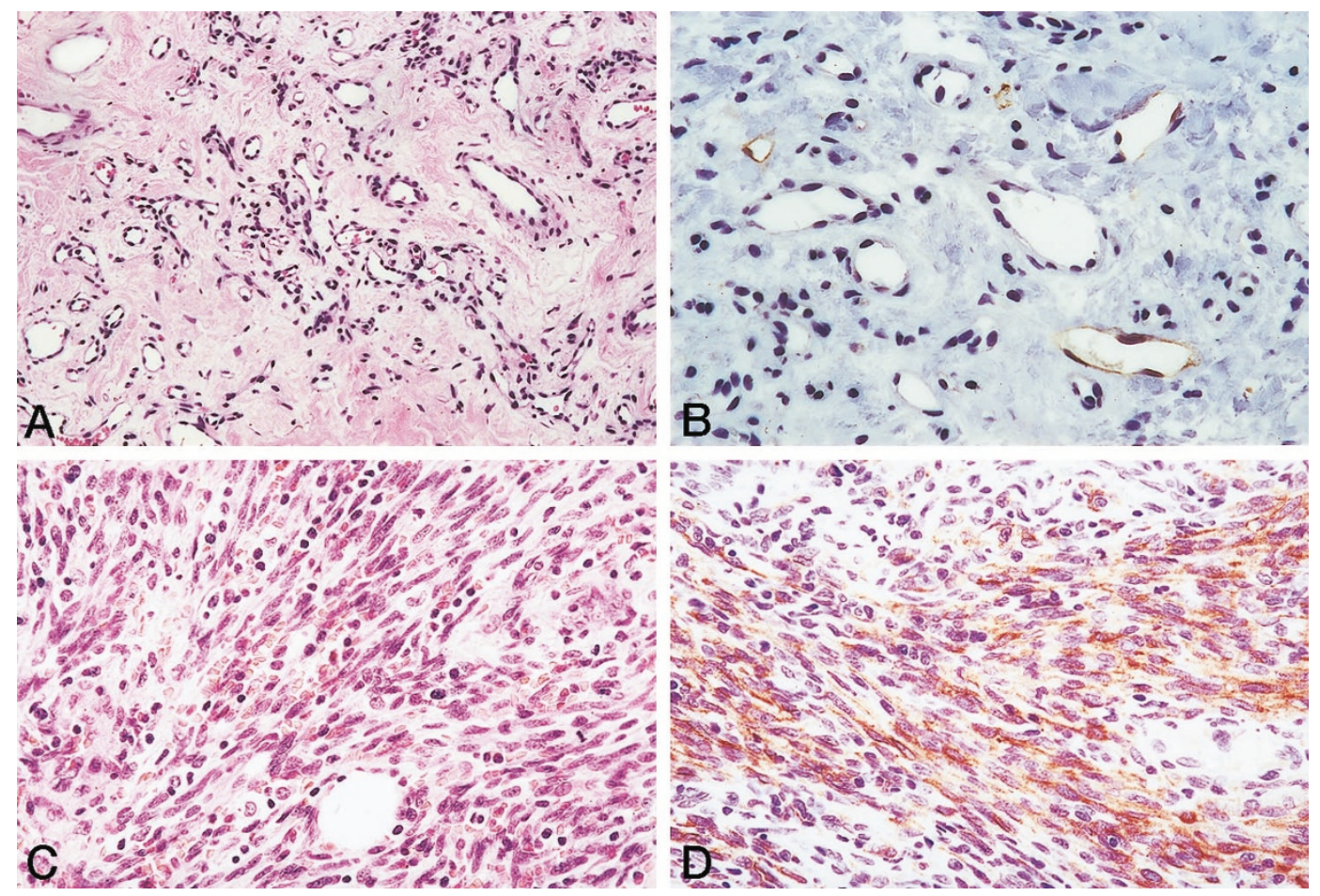

FIGURE 1. A low power view of a typical case of capillary hemangioma, showing the VEGFR-3-negative tumor vessels and the positive normal lymphatics (A and B). In contrast, all but one case of Kaposi's sarcoma were intensely VEGFR-3 positive (C and D). (A, hematoxylin and eosin, 100×; B, anti-VEGFR-3, 200×; C, hematoxylin and eosin, 200×; D, anti-VEGFR-3, 200×).

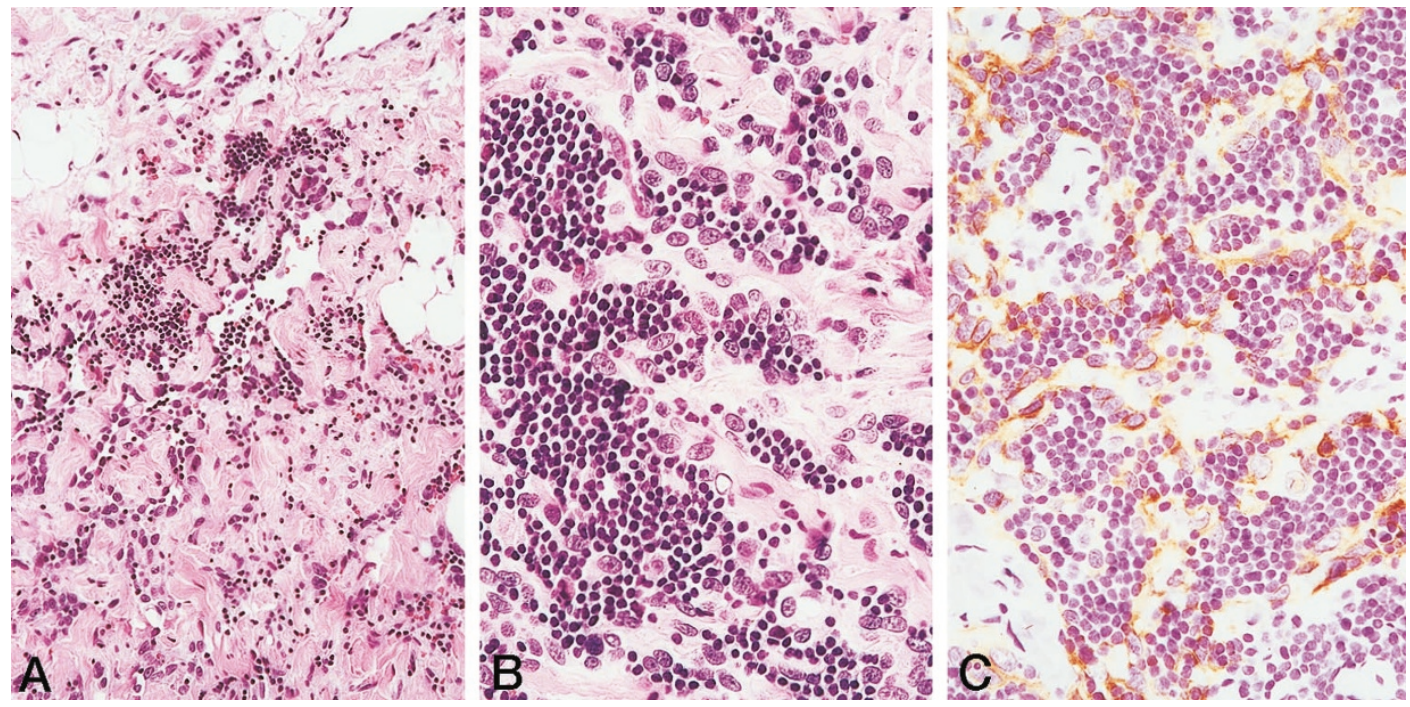

FIGURE 2. Low (A) and medium (B) power views of an angiosarcoma, not associated with lymphedema, characterized by a very prominent lymphocytic inflammatory infiltrate. The three angiosarcomas with these features were all strongly VEGFR-3 positive (C). (A, hematoxylin and eosin, 40×; B, hematoxylin and eosin, 200×; C, anti-VEGFR-3, 200×).

embedded and frozen sections from eight and three cases of KS, respectively (4). Subsequently, Lymboussaki and co-workers (10) found VEGFR-3 expression in six cases of paraffin-embedded dermal lymphangiomatosis and three cryosectioned intramuscular hemangiomas but not in three paraffinembedded capillary hemangiomas.

We examined the immunohistochemical expression of VEGFR-3 in 70 cases of paraffin-embedded vascular tumors, including 25 cases of KS. Our re- sults strongly affirm the superb sensitivity of antibodies to VEGFR-3 for KS; the spindle cells in 24 of 25 cases of KS, at all stages of development and including metastatic lesions, were strongly positive for VEGFR-3. The sensitivity of anti-VEGFR-3 in paraffin-embedded sections $(96 \%)$ compares very favorably to that reported for CD31 (89\%) (5), CD34 (93\%) (19), and vWF (64\%) (20). Our findings of slightly greater sensitivity for CD31 (93\%) and vWF (79\%) may be the result of our uniform use of 


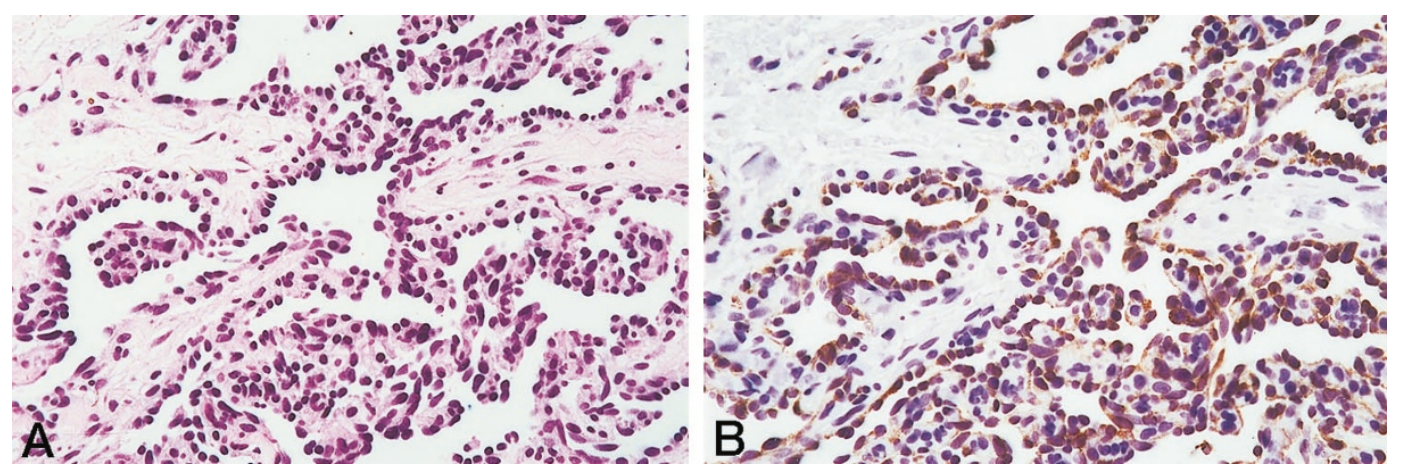

FIGURE 3. Two VEGFR-3-positive angiosarcomas were characterized by "hobnailed" endothelial cells and endovascular tufting, as is seen in Dabska's tumor, but showed in addition infiltrative and irregular growth. (A, hematoxylin and eosin, 200×; B, anti-VEGFR-3, 200×).
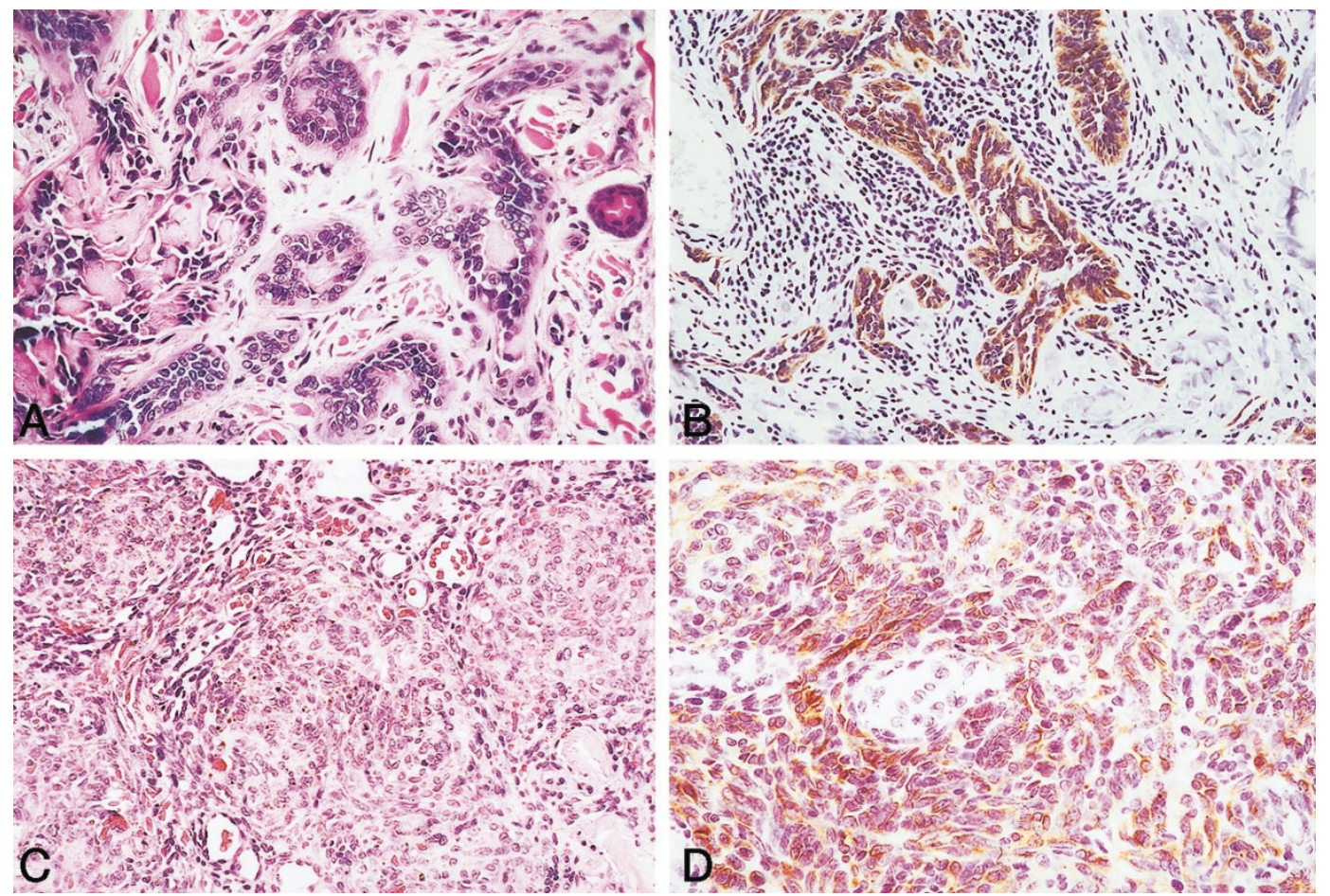

FIGURE 4. All cases of both Dabska's tumor (A and B) and kaposiform hemangioendothelioma (C and D) strongly expressed VEGFR-3. (A and C, hematoxylin and eosin, 200×; B, anti-VEGFR-3, 100×; D, anti-VEGFR-3, 200×).

heat-induced epitope retrieval. Furthermore, the immunoreactivity was generally present in more than $50 \%$ of the tumor cells, with a very low level of nonspecific "background" staining. In all cases, small, normal vascular spaces that were believed morphologically to be consistent with either lymphatics or small venules were also positive; endothelia in clearly identifiable arteries and veins were always negative. Significantly, we did not observe any other normal structure or cell to be positive for VEGFR-3. This is in strong contrast to CD34, which is positive in normal dendritic interstitial cells surrounding blood vessels and nerves and in a variety of nonvascular tumors, including dermatofibrosarcoma protuberans, gastrointestinal stromal tumors, and solitary fibrous tumors (19). CD31 may also be expressed by nonendothelial cells, including mono- cytes/macrophages $(21,22)$, very rare carcinomas (23), and epithelioid sarcomas (24). We are in the process of studying VEGFR-3 expression in nonvascular tumors but see no reason to believe that it will be present in more than extremely rare cases.

There is debate in the literature, as well as a great deal of contradictory evidence, about whether the cells of KS show lymphatic or vascular endothelial differentiation. Some authors have favored lymphatic endothelial differentiation, based on ultrastructural findings of discontinuous basal lamina and the absence of dendritic pericytes (25), and immunohistochemical findings such as absent or only weak vWF and Ulex europaeus lectin expression $(2,6,26)$. Other authors have claimed blood vessel endothelial differentiation in KS, on the basis of immunohistochemical expression of vWF in fro- 
zen sections $(7,9)$, the demonstration of uniform laminin investment of the spindle cells (2) or the supposed absence of CD31 on both KS and lymphatic endothelium (27). In attempting to unravel these often contradictory claims, it is important to note that whereas early studies suggested that vWF, CD34, and CD31 were expressed only by blood vessel endothelium, more recent studies have shown weaker but demonstrable levels of all three antigens in lymphatic endothelium $(4,10,28)$. This implies that the presence or absence of these antigens is not an absolute indication of differentiation in a vascular tumor. Our finding of VEGFR-3 expression in almost all cases of KS may support the concept that KS shows lymphatic endothelial differentiation. An alternative is that KS may recapitulate an immature endothelial cell phenotype, thereby explaining VEGFR-3 positivity (18).

We had hoped that the finding of VEGFR-3 expression might be useful in separating KS from other vascular tumors with which it may be confused, including spindled variants of AS and kaposiform hemangioendothelioma. Unfortunately, among vascular tumors, VEGFR-3 expression is not specific for KS. We were able to find VEGFR-3 expression in 8 of 16 AS, with a staining intensity comparable to KS. It is interesting that of these eight positive cases, six were characterized by highly unusual appearance. Three displayed a striking lymphocytic infiltrate, although none arose in the setting of lymphedema. Two were characterized by small cuboidal endothelial cell reminiscent of those of the Dabska tumor yet grew in an infiltrative and irregular pattern similar to a conventional AS. Another was a highly spindled AS, with a prominent lymphocytic infiltrate, arising from the peritoneal surface, which in many areas out of context resembled KS. One could therefore mount a reasonable argument that these AS represent one or more distinct subsets perhaps linked by lymphatic endothelial differentiation. That some AS might display lymphatic differentiation was implied by the early term lymphangiosarcoma. This term was later supplanted by the generic angiosarcoma because of the inability to separate precisely lymphatic from capillary vascular endothelial differentiation by light microscopy. However, the diverse clinical settings in which AS develop (cf. postirradiation, lymphedema associated), buttressed by the results of our study, lend support to the idea that AS comprise several phenotypic and possibly biologic subsets. None of the AS in the current study were known to have been associated with chronic lymphedema or prior irradiation.

We have also found uniform expression of VEGFR-3 in two rare pediatric vascular tumors of intermediate malignancy, kaposiform hemangioendothelioma and Dabska tumor. This finding is notable because both of these tumors have been postulated to show lymphatic endothelial differentiation, on the basis of their characteristic infiltration by lymphocytes $(29,30)$, the association of the former with lymphangiomatosis $(29$, 31 ), and their reported usual absence of vWF expression $(29,31)$. We observed vWF expression in $80 \%$ of kaposiform hemangioendothelioma and all Dabska tumors; again, this difference may be the result of our use of heat-induced epitope retrieval.

We also saw expression of VEGFR-3 in only 2 of 13 cases of capillary hemangioma, in general agreement with the previously reported results of Lymboussaki et al. (10). We found VEGFR-3 positivity in only one of the three intra-abdominal lymphangiomas, as compared with the uniform positivity previously noted in dermal lymphangiomatosis (10); possibly this may reflect lower production of this protein in intra-abdominal tumors as compared with dermal ones.

In conclusion, we have shown immunohistochemical expression of VEGFR-3 in 96\% of cases of $\mathrm{KS}$, all cases of kaposiform hemangioendothelioma and Dabska tumor, and a significant subset of AS. The sensitivity of anti-VEGFR-3 for KS is higher than that of the routinely used vascular markers CD31, CD34, and vWF and suggests a valuable role for this antibody in the diagnosis of difficult cases of KS. In particular, this antibody could serve to distinguish various fibrovascular proliferations (e.g., stasis changes, chronic ulcers with surrounding reactive fibrosis) from KS. Our findings also support the concept that the KS, kaposiform hemangioendothelioma, and the Dabska tumor have a lymphatic endothelial phenotype. AS expressing this receptor protein may represent tumors displaying lymphatic differentiation. Whether these phenotypic differences will translate into biologic ones requires additional study.

\section{REFERENCES}

1. Scully PA, Steinman HK, Kennedy C, Trueblood K, Frisman DM, Voland JR. AIDS-related Kaposi's sarcoma displays differential expression of endothelial surface antigens. Am J Pathol 1988;130(2):244-51.

2. Dictor M, Andersson C. Lymphaticovenous differentiation in Kaposi's sarcoma: cellular phenotypes by stage. Am J Pathol 1988;130(2):411-7 [published erratum appears in 133(2):226].

3. Zhang YM, Bachmann S, Hemmer C, van Lunzen J, von Stemm A, Kern P, et al. Vascular origin of Kaposi's sarcoma: expression of leukocyte adhesion molecule-1, thrombomodulin, and tissue factor. Am J Pathol 1994;144(1):51-9.

4. Jussila L, Valtola R, Partanen TA, Salven P, Heikkila P, Matikainen MT, et al. Lymphatic endothelium and Kaposi's sarcoma spindle cells detected by antibodies against the vascular endothelial growth factor receptor-3. Cancer Res 1998; 58(8):1599-604.

5. Hoerl HD, Goldblum JR. Immunoreactivity patterns of CD31 and CD68 in 28 cases of Kaposi's sarcoma: evidence supporting endothelial differentiation in the spindle cell component. Appl Immunohistochem 1997;5(3):173-8. 
6. Kostianovsky M, Lamy Y, Greco MA. Immunohistochemical and electron microscopic profiles of cutaneous Kaposi's sarcoma and bacillary angiomatosis. Ultrastruct Pathol 1992; 16(6):629-40.

7. Rutgers JL, Wieczorek R, Bonetti F, Kaplan KL, Posnett DN, Friedman-Kien AE, et al. The expression of endothelial cell surface antigens by AIDS-associated Kaposi's sarcoma: evidence for a vascular endothelial cell origin. Am J Pathol 1986;122(3):493-9.

8. Dorfman RF, Wood GS, Beckstead JH. Histogenesis of Kaposi's sarcoma. Kaposi's sarcoma: pathophysiology and clinical management. Ziegler JL, Dorfman RF, eds. New York, Marcel Dekker, p 1988.

9. Facchetti F, Lucini L, Gavazzoni R, Callea F. Immunomorphological analysis of the role of blood vessel endothelium in the morphogenesis of cutaneous Kaposi's sarcoma: a study of 57 cases. Histopathology 1988;12(6):581-93.

10. Lymboussaki A, Partanen TA, Olofsson B, Thomas-Crusells J, Fletcher CD, de Waal RM, et al. Expression of the vascular endothelial growth factor $C$ receptor VEGFR-3 in lymphatic endothelium of the skin and in vascular tumors. Am J Pathol 1998;153(2):395-403.

11. Miettinen M, Lindenmayer AE, Chaubal A. Endothelial cell markers CD31, CD34, and BNH9 antibody to $\mathrm{H}$ - and Yantigens: evaluation of their specificity and sensitivity in the diagnosis of vascular tumors and comparison with von Willebrand factor. Mod Pathol 1994;7(1):82-90.

12. Suster S, Nascimento AG, Miettinen M, Sickel JZ, Moran CA. Solitary fibrous tumors of soft tissue: a clinicopathologic and immunohistochemical study of 12 cases. Am J Surg Pathol 1995;19(11):1257-66.

13. Suster S, Fisher C. Immunoreactivity for the human hematopoietic progenitor cell antigen (CD34) in lipomatous tumors. Am J Surg Pathol 1997;21(2):195-200.

14. Weiss SW, Nickoloff BJ. CD-34 is expressed by a distinctive cell population in peripheral nerve, nerve sheath tumors, and related lesions [see comments]. Am J Surg Pathol 1993; 17(10):1039-45.

15. Ferrara N, Davis-Smyth T. The biology of vascular endothelial growth factor. Endocr Rev 1997;18(1):4-25.

16. Jeltsch M, Kaipainen A, Joukov V, Meng X, Lakso M, Rauvala $\mathrm{H}$, et al. Hyperplasia of lymphatic vessels in VEGF-C transgenic mice. Science 1997;276(5317):1423-5 [published erratum appears in 277(5325):463].

17. Oh SJ, Jeltsch MM, Birkenhager R, McCarthy JE, Weich HA, Christ B, et al. VEGF and VEGF-C: specific induction of angiogenesis and lymphangiogenesis in the differentiated avian chorioallantoic membrane. Dev Biol (Orlando) 1997; 188(1):96-109.

18. Kaipainen A, Korhonen J, Mustonen T, van Hinsbergh VW, Fang GH, Dumont D, et al. Expression of the fms-like ty- rosine kinase 4 gene becomes restricted to lymphatic endothelium during development. Proc Natl Acad Sci U S A 1995;92(8):3566-70.

19. van de Rijn M, Rouse R. CD34: a review. Appl Immunohistochem 1994;2(2):71-80.

20. Sankey EA, More L, Dhillon AP. QBEnd/10: a new immunostain for the routine diagnosis of Kaposi's sarcoma [see comments]. J Pathol 1990;161(3):267-71.

21. Watt SM, Gschmeissner SE, Bates PA. PECAM-1: its expression and function as a cell adhesion molecule on hemopoietic and endothelial cells. Leuk Lymphoma 1995;17(3-4): $229-44$.

22. Szekanecz Z, Haines GK, Harlow LA, Shah MR, Fong TW, Fu $\mathrm{R}$, et al. Increased synovial expression of the adhesion molecules CD66a, CD66b, and CD31 in rheumatoid and osteoarthritis. Clin Immunol Immunopathol 1995;76(2):180-6.

23. De Young BR, Frierson HF, Jr., Ly MN, Smith D, Swanson PE. CD31 immunoreactivity in carcinomas and mesotheliomas. Am J Clin Pathol 1998;110(3):374-7.

24. Smith ME, Brown JI, Fisher C. Epithelioid sarcoma: presence of vascular-endothelial cadherin and lack of epithelial cadherin. Histopathology 1998;33(5):425-31.

25. McNutt NS, Fletcher V, Conant MA. Early lesions of Kaposi's sarcoma in homosexual men: an ultrastructural comparison with other vascular proliferations in skin. Am J Pathol 1983; 111(1):62-77.

26. Beckstead JH, Wood GS, Fletcher V. Evidence for the origin of Kaposi's sarcoma from lymphatic endothelium. Am J Pathol 1985;119(2):294-300.

27. Parums DV, Cordell JL, Micklem K, Heryet AR, Gatter KC, Mason DY. JC70: a new monoclonal antibody that detects vascular endothelium associated antigen on routinely processed tissue sections. J Clin Pathol 1990;43(9):752-7.

28. Sauter B, Foedinger D, Sterniczky B, Wolff K, Rappersberger $\mathrm{K}$. Immunoelectron microscopic characterization of human dermal lymphatic microvascular endothelial cells: differential expression of CD31, CD34, and type IV collagen with lymphatic endothelial cells vs blood capillary endothelial cells in normal human skin, lymphangioma, and hemangioma in situ. J Histochem Cytochem 1998;46(2):165-76.

29. Zukerberg LR, Nickoloff BJ, Weiss SW. Kaposiform hemangioendothelioma of infancy and childhood: an aggressive neoplasm associated with Kasabach-Merritt syndrome and lymphangiomatosis. Am J Surg Pathol 1993;17(4):321-8.

30. Dabska M. Malignant endovascular papillary angioendothelioma of the skin in childhood: clinicopathologic study of 6 cases. Cancer 1969;24(3):503-10.

31. Mentzel T, Mazzoleni G, Dei Tos AP, Fletcher CD. Kaposiform hemangioendothelioma in adults: clinicopathologic and immunohistochemical analysis of three cases. Am J Clin Pathol 1997;108(4):450-5. 